\title{
Dipole Moments of Dimethylsiloxane Oligomers and Poly(dimethylsiloxane)
}

\author{
C. Sutton and J. E. Mark \\ Department of Chemistry, University of Michigan, Ann Arbor, Michigan 48104
}

(Received 8 February 1971)

\begin{abstract}
Dielectric constants at $20,30,40$, and $50^{\circ} \mathrm{C}$ have been determined for pure liquid dimethylsiloxane oligomers $\left(\mathrm{CH}_{3}\right)_{3} \mathrm{Si}-\left[\mathrm{OSi}\left(\mathrm{CH}_{3}\right)_{2}\right]_{x} \mathrm{OSi}\left(\mathrm{CH}_{3}\right)_{3}$ having chain lengths corresponding to $x=3,5,7$, and 9 . Mean-square dipole moments $\left\langle\mu^{2}\right\rangle$, calculated from the Onsager equation, are in good agreement with predicted values based on a rotational isomeric state model with neighbor dependence and chain conformational energies obtained in an independent analysis of the random-coil dimensions of such chains. In addition, the observed temperature coefficients of $\left\langle\mu^{2}\right\rangle$ are in qualitative agreement with calculated results for the stated range of $x$. An experimental value of the ratio $\left\langle\mu^{2}\right\rangle / n m^{2}$ (where $n$ is the number of bond dipoles, each of magnitude $m$ ) in the limit of large $x$ is estimated from published data on the pure liquid polymer at $25^{\circ} \mathrm{C}$. This tentative result is in fair agreement with theory; agreement is improved if the large specific solvent effect previously observed for this polymer is interpreted as being primarily due to the effect of the dielectric constant of the medium on the Coulombic contribution to the conformational energy.
\end{abstract}

\section{INTRODUCTION}

The configuration-dependent properties of linear dimethylsiloxane chains $\left(\mathrm{CH}_{3}\right)_{3} \mathrm{Si}\left[\mathrm{OSi}\left(\mathrm{CH}_{3}\right)_{2}\right]_{x} \mathrm{OSi}\left(\mathrm{CH}_{3}\right)_{3}$ have been intensively studied by a number of workers. For example, experimental studies of these molecules have included measurements of their random-coil dimensions, ${ }^{1,2}$ stress-optical coefficients, ${ }^{3}$ ring-chain equilibrium constants, ${ }^{4,5}$ and dipole moments. ${ }^{6-8}$ The experimental results to date have all been adequately interpreted ${ }^{3,5,9-11}$ by statistical mechanical calculations based on a rotational isomeric state model of these chains which takes into account neighbor interactions. ${ }^{12}$

The dipole moment has proved to be a particularly useful quantity for characterizing the configuration of a chain molecule which contains polar groups because it, unlike several other configuration-dependent properties, can readily be measured over the entire range of molecular weight. The most reliable experimental study of dipole moments of several dimethylsiloxane oligomers at a number of temperatures is that of Dasgupta and Smyth, ${ }^{8}$ who showed that previous disagreement between experimental results was due to approximations made regarding the atomic polarization, a significant part of the total polarization observed for these chain molecules. Their values of the dipole moments were shown to be in agreement with theoretical results, ${ }^{11}$ but the range of chain length experimentally investigated was relatively small $(x=1,2$, 3 , and 5) and the results were not of sufficient precision to permit comparison between experimental and theoretical values of the temperature coefficient of the dipole moment. It is the purpose of the present study to obtain additional experimental values of the dipole moments of dimethylsiloxane chains at a number of temperatures, thereby providing a more definitive comparison of theory and experiment.

\section{EXPERIMENTAL MEASUREMENTS}

Dimethylsiloxane oligomers having chain lengths corresponding to $x=3,5,7$, and 9 were obtained in a state of purity exceeding $99 \%$; poly (dimethylsiloxane) was obtained as an unfractionated material of molecular weight $\sim 20000(x \cong 270)$ from which low-molecularweight species had been removed by heating under vacuum. ${ }^{13}$ The densities of each oligomer at $20,30,40$, and $50^{\circ} \mathrm{C}$ were determined by use of a pycnometer, in a constant temperature bath controlled to $\pm 0.01^{\circ} \mathrm{C}$. Indices of refraction $n$ at 436 and $589 \mathrm{~m} \mu$, and at each of the stated temperatures, were measured for both the oligomers and polymer by means of a precision Bausch \& Lomb refractometer of the Abbe design.

The dielectric constant apparatus consisted of a DM01 dipole meter ${ }^{14}$ operating at a fixed frequency of $2.0 \mathrm{MHz}$ and a cylindrical, gold-plated, thermostatted cell having a sample volume of approximately $4 \mathrm{~cm}^{3}$. Calibration of the apparatus was carried out at each temperature using benzene, cyclohexane, and carbon tetrachloride. These materials (Matheson, Coleman, and Bell; Chromatoquality) had a purity exceeding 99 mole \% as received and were further purified by means of Linde type $4 \mathrm{~A}$ molecular sieves. Since the dipole moments of the oligomers are quite low, it is not necessary to study solutions of these materials in a nonpolar solvent ${ }^{8}$; dielectric constant measurements were therefore made directly on the pure, undiluted oligomers at each of the four temperatures. At the relatively low frequency of $2 \mathrm{MHz}$, one obtains to a very good approximation the so-called "static" dielectric constant $\epsilon_{0}$.

\section{EXPERIMENTAL RESULTS}

The experimentally determined densities of the four oligomers are given in the third column of Table I; included also is a reported value of the density at $25^{\circ} \mathrm{C}$ of a sample of poly(dimethylsiloxane) having $x \cong 300$. Molar volumes $V$ were obtained from these densities and molecular weights $M$ calculated from the structural formulas by $M\left(\right.$ gram $\left.\mathrm{mole}^{-1}\right)=162.4+$ $74.17 x$. Values of the indices of refraction in the limit of infinite wavelength, $n_{\infty}$, were obtained from the 
TABLE I. Summary of data and results, $\left(\mathrm{CH}_{3}\right)_{3} \mathrm{Si}\left[\mathrm{OSi}\left(\mathrm{CH}_{3}\right)_{2}\right]_{x} \mathrm{OSi}\left(\mathrm{CH}_{3}\right)_{3}$ chains.

\begin{tabular}{|c|c|c|c|c|c|c|c|c|}
\hline \multirow[b]{2}{*}{$x$} & \multirow{2}{*}{$\begin{array}{c}T \\
\left({ }^{\circ} \mathrm{C}\right)\end{array}$} & \multirow{2}{*}{$\begin{array}{c}d \\
\left(\mathrm{~g} / \mathrm{cm}^{3}\right)\end{array}$} & \multirow[b]{2}{*}{$n_{\infty}$} & \multirow[b]{2}{*}{$\epsilon_{\infty}$} & \multirow[b]{2}{*}{$\epsilon_{0}$} & \multirow{2}{*}{$\begin{array}{c}\mu \\
(\mathrm{D})\end{array}$} & \multicolumn{2}{|c|}{$\left\langle\mu^{2}\right\rangle / n m^{2}$} \\
\hline & & & & & & & Exptl & Theoret \\
\hline \multirow[t]{4}{*}{3} & 20 & 0.8748 & 1.3807 & 2.215 & 2.476 & 0.949 & 0.313 & 0.311 \\
\hline & 30 & 0.8648 & 1.3766 & 2.198 & 2.445 & $0.94_{g}$ & 0.313 & 0.304 \\
\hline & 40 & 0.8550 & 1.3727 & 2.182 & 2.411 & $0.93_{8}$ & 0.305 & 0.295 \\
\hline & 50 & 0.8448 & 1.3687 & 2.166 & 2.386 & $0.94_{3}$ & 0.309 & 0.291 \\
\hline \multirow[t]{4}{*}{5} & 20 & 0.9014 & 1.3846 & 2.262 & 2.589 & 1.21 & 0.341 & 0.300 \\
\hline & 30 & 0.8917 & 1.3803 & 2.243 & 2.552 & 1.21 & 0.340 & 0.294 \\
\hline & 40 & 0.8822 & 1.3766 & 2.227 & 2.489 & 1.15 & 0.305 & 0.290 \\
\hline & 50 & 0.8725 & 1.3729 & 2.211 & 2.475 & 1.19 & 0.323 & 0.287 \\
\hline \multirow[t]{4}{*}{7} & 20 & 0.9182 & 1.3870 & 2.286 & 2.645 & 1.41 & 0.347 & 0.282 \\
\hline & 30 & 0.9084 & 1.3823 & 2.266 & 2.601 & 1.41 & 0.343 & 0.279 \\
\hline & 40 & 0.8990 & 1.3788 & 2.250 & 2.555 & 1.38 & 0.329 & 0.277 \\
\hline & 50 & 0.8891 & 1.3749 & 2.233 & 2.519 & 1.37 & 0.325 & 0.274 \\
\hline \multirow[t]{4}{*}{9} & 20 & 0.9265 & 1.3880 & 2.299 & 2.669 & 1.57 & 0.343 & 0.272 \\
\hline & 30 & 0.9170 & 1.3838 & 2.280 & 2.627 & 1.56 & 0.340 & 0.270 \\
\hline & 40 & 0.9076 & 1.3799 & 2.264 & 2.582 & 1.54 & 0.329 & 0.268 \\
\hline & 50 & 0.8979 & 1.3761 & 2.247 & 2.548 & 1.53 & 0.327 & 0.267 \\
\hline 300 & 25 & $0.970^{\mathrm{a}}$ & 1.3917 & 2.359 & $2.785^{\mathrm{a}}$ & 8.44 & 0.329 & 0.231 \\
\hline
\end{tabular}

a Taken from Ref. 7 .

experimental values of $n$ at two wavelengths using the approximate equation suggested by Smyth $^{8,15}$; they are given in the following column of the table. The electronic polarization $P_{E}$ was then calculated from

$$
P_{E}=V\left(n_{\infty}^{2}-1\right) /\left(n_{\infty}^{2}+2\right),
$$

and the atomic polarization $P_{A}$ was obtained from the relationship $P_{A}$ (cubic centimeters mole $^{-1}$ ) $=7.81+$ $5.66 x$, which fits the experimental results of Dasgupta and Smyth ${ }^{8}$ to within a few tenths of a percent. The sum of these two polarizations was used to calculate the infinite frequency, or optical, dielectric constant $\epsilon_{\infty}$ from

$$
P_{E}+P_{A}=V\left(\epsilon_{\infty}-1\right) /\left(\epsilon_{\infty}+2\right) \text {. }
$$

Values of $\epsilon_{\infty}$ thus obtained are given in Column 5 of the table, and values of the experimentally determined low-frequency dielectric constant for both the oligomers and polymer ${ }^{7}$ are given in Column 6. Mean-square dipole moments $\left\langle\mu^{2}\right\rangle$ were then calculated from the Onsager equation ${ }^{16}$ in which $n^{2}$ has been replaced by $\epsilon_{\infty},{ }^{8}$

$$
\left\langle\mu^{2}\right\rangle=\left(9 \epsilon^{\prime} k T V / 4 \pi N\right)\left[\left(\epsilon_{0}-\epsilon_{\infty}\right)\left(2 \epsilon_{0}+\epsilon_{\infty}\right) / \epsilon_{0}\left(\epsilon_{\infty}+2\right)^{2}\right] \text {. }
$$

In this equation, $\boldsymbol{\epsilon}^{\prime}$ is permittivity of empty space, $k$ is the Boltzmann constant, and $N$ is the Avogadro number. Values of the square root of $\left\langle\mu^{2}\right\rangle$, in debyes (D), are given in Column 7 of the table. A more useful quantity is the mean-square dipole moment relative to $\mathrm{nm}^{2}$, where $n=2 x+2$ is the number of $\mathrm{Si}-\mathrm{O}$ and $\mathrm{O}-\mathrm{Si}$ bond dipoles in the chain and $m^{2}$ is the square of their moments, $\pm 0.60 \mathrm{D} .{ }^{11}$ The dipole moment ratio $\left\langle\mu^{2}\right\rangle / \mathrm{nm}^{2}$ thus defined represents the factor by which the meansquare moment of the actual chain departs from that of the same chain in the idealization that all of the skeletal bonds are freely jointed. The experimental values of this ratio are tabulated in Column 8 of the table.

\section{THEORETICAL RESULTS}

In an earlier study, ${ }^{11}$ a rotational isomeric state model with neighbor dependence was used to calculate values of $\left\langle\mu^{2}\right\rangle / \mathrm{nm}^{2}$ for dimethylsiloxane chains over the entire range of chain length. Structural quantities employed in these calculations were bond angles about $\mathrm{Si}$ and $\mathrm{O}$ atoms of $110^{\circ}$ and $143^{\circ}$, respectively, and $\mathrm{Si}-\mathrm{O}$ and $\mathrm{O}-\mathrm{Si}$ bond moments of $m= \pm 0.60 \mathrm{D}$, respectively. The required conformational energies were taken without modification from a previous study of highmolecular-weight poly(dimethylsiloxane), where they were obtained by comparison of theoretical and experimental values of the unperturbed, random-coil dimensions and their temperature coefficient, ${ }^{1,2,9}$ Specifically, these results are an energy $E_{\sigma}$ of $0.85 \mathrm{kcal} \mathrm{mole}^{-1}$ for gauche states, relative to trans states, about $\mathrm{Si}-\mathrm{O}$ and $\mathrm{O}-\mathrm{Si}$ bonds, and an energy $E_{\omega}=1.05 \mathrm{kcal} \mathrm{mole}^{-1}$ which accounts for the additional interaction occurring when two consecutive gauche states of opposite sign bring into proximity oxygen atoms separated by four skeletal bonds. Statistical weights of these conformations were expressed as Boltzmann factors in the corresponding 
energies: $\sigma=\exp \left(-E_{\sigma} / R T\right)$ and $\omega=\exp \left(-E_{\omega} / R T\right)$, where $R$ is the gas constant. These statistical weights and the structural data given above were then used to calculate theoretical values of $\left\langle\mu^{2}\right\rangle / n m^{211,17}$ by means of the statistical mechanical theory of chain configuration as developed by Flory and co-workers. ${ }^{12}$ The results which pertain to the values of chain length and temperature experimentally investigated herein are shown in the last column of the table.

\section{DISCUSSION}

Some of the experimental results obtained in the present study and summarized in the table may be compared with the results of Dasgupta and Smyth ${ }^{8}$; they also studied the oligomers corresponding to $x=3$ and 5 , over a similar temperature range. The experimental values of the dipole-moment ratios for these two values of $x$ agree to within a few percent. The average difference between the theoretical and present experimental values of $\left\langle\mu^{2}\right\rangle / n m^{2}$ for all four oligomers is approximately $12 \%$ (relative to the experimental values); the average difference between values of $\mu$ itself would be approximately $6 \%$. As pointed out previously, ${ }^{11}$ the relatively low values $(0.2-0.3)$ found for $\left\langle\mu^{2}\right\rangle / \mathrm{nm}^{2}$ for these molecules is due to the fact that in the energetically preferred conformations of the chain, the bond dipole vectors are more frequently in opposed, or partially opposed, orientations than in the freely jointed chain.

In short dimethylsiloxane chains, the transitions from trans to gauche states about $\mathrm{Si}-\mathrm{O}$ and $\mathrm{O}-\mathrm{Si}$ bonds caused by an increase in temperature increase the attenuation of $\left\langle\mu^{2}\right\rangle$ resulting from the opposition of bond dipole vectors, and thus the temperature coefficient of $\left\langle\mu^{2}\right\rangle$ is predicted to be negative. Specifically, $d \ln \left\langle\mu^{2}\right\rangle / d T$ for oligomers in the range of $x$ studied is expected to be the order of $-1 \times 10^{-3} \mathrm{deg}^{-1} .^{11}$ For the temperature range employed, this would result in a change in $\left\langle\mu^{2}\right\rangle / n m^{2}$ of only approximately $3 \%$, or 0.01 unit; since this is not much larger than the probable precision of the measurements, only a qualitative comparison of theoretical and experimental values of this coefficient is possible. Since, as shown in the table, the experimental values of $\left\langle\mu^{2}\right\rangle / n m^{2}$ do appear to decrease by approximately $0.01 \mathrm{D}$ upon increase of the temperature from 20 to $50^{\circ} \mathrm{C}$, the theoretical predictions seem to be qualitatively confirmed in the case of these oligomers.

If the tentative value of $\left\langle\mu^{2}\right\rangle / n m^{2}$ at $x=300$ estimated from the data of Baker et al..$^{7}$ is correct, the difference between theoretical and experimental values of this ratio in the limit of large $x$ would amount to almost $30 \%$. Part of this difference could be due to inaccuracies in the long extrapolation required to estimate the atomic polarization at large values of $x$. A more general difficulty could be the effect of the dielectric constant of the medium on the conformational energy of the chain.
Such an effect has, in fact, been suggested ${ }^{9}$ as the basis for the significant difference observed in the unperturbed dimensions of poly(dimethylsiloxane) chains in solvents of very different dielectric constants, at essentially the same temperature. ${ }^{2}$ Specifically, light scattering measurements in a solvent mixture of low dielectric constant gave a value of the mean-square unperturbed dimensions $22 \%$ larger than those obtained in methylethyl ketone, which has a large dielectric constant, approximately $19 .{ }^{18}$ According to the theoretical analysis, ${ }^{9}$ a decrease in the dielectric constant of the medium should increase the effect of Coulombic interactions in such a way as to suppress the number of compact conformations (primarily through decrease of $E_{\sigma}$ ). The increase in the unperturbed dimensions with decrease in the dielectric constant of the medium was thus qualitatively explained.

The conformational energies used in the present calculations were obtained ${ }^{9}$ by analysis of the unperturbed dimensions of poly (dimethylsiloxane) chains in methyl ethyl ketone, since insufficient experimental results were available in the mixed solvent system. ${ }^{2}$ Since the experimental values of $\left\langle\mu^{2}\right\rangle / n m^{2}$ were obtained in a medium of low dielectric constant $(\sim 2)$, the theoretical values of this ratio should be adjusted to account for changes in conformational energy. As pointed out earlier ${ }^{9}$ it is not possible at present to carry out such a correction quantitatively, but the qualitative effect can easily be predicted. Suppression of compact conformations of the chain by decrease in the dielectric constant of the medium should increase the values of the predicted dipole moments because of the likelihood of at least partially opposed bond dipoles in such conformations. Proper account of the effect of the dielectric constant of the medium would therefore be expected to improve the agreement between theoretical and experimental values of the dipole moments of poly (dimethylsiloxane) chains. The magnitude of this effect over the entire range of chain length is presently under investigation.

\section{ACKNOWLEDGMENT}

It is a pleasure to acknowledge the financial support provided by Grant GP-16028 from the National Science Foundation.

${ }^{1} \mathrm{~J}$. E. Mark and P. J. Flory, J. Am. Chem. Soc. 86, 138 (1964). 2 V. Crescenzi and P. J. Flory, J. Am. Chem. Soc. 86, 141 (1964)

${ }^{3}$ K. Nagai and T. Ishikawa, J. Chem. Phys. 45, 3128 (1966). ${ }^{4}$ J. F. Brown, Jr., and G. M. J. Slusarczuk, J. Am. Chem. Soc. 87, 931 (1965).

5 J. A. Semlyen and P. V. Wright, Polymer 10, 543 (1969).

${ }^{6}$ R. O. Sauer and D. J. Mead, J. Am. Chem. Soc. 68, 1794 (1946)

${ }_{7}$ E. B. Baker, A. J. Barry, and M. J. Hunter, Ind. Eng. Chem. 38, 1117 (1946).

8 S. Dasgupta and C. P. Smyth, J. Chem. Phys. 47, 2911 (1967).

${ }_{9}$ P. J. Flory, V. Crescenzi, and J. E. Mark, J. Am. Chem. Soc. $86,146(1964)$.

${ }_{10}$ P. J. Flory and J. A. Semlyen, J. Am. Chem. Soc. 88, 3209 (1966). 
11 J. E. Mark, J. Chem. Phys. 49, 1398 (1968)

${ }_{12}$ P. J. Flory, Statistical Mechanics of Chain Molecules (Interscience, New York, 1969).

${ }^{13}$ These samples were generously provided by Dr. T. Williams of the Union Carbide Corp. and Dr. J. Saam of the Dow-Corning Corp.

${ }^{14}$ Constructed by the Wissenschaftlich-Technische Werkstätten, GMBH, Weilheim Oberbayern, Germany; available in the United States from the Kahl Scientific Instrument Co., El Cajon, Calif.
${ }^{15}$ C. P. Smyth, Dielectric Behavior and Structure (McGraw-Hill, New York, 1955), p. 405.

${ }^{16}$ L. Onsager, J. Am. Chem. Soc. 58, 1486 (1936).

${ }^{17}$ There are two errors in the description of this calculation in Ref. 11: a unit matrix of order three should replace the last statistical-weight matrix $U_{a}$ appearing in Fq. (6) and should also replace the $U_{a}$ appearing in the last generator matrix $G_{a}$ in Eq. (8).

${ }_{18}$ American Institute of Physics IIandbook, edited by D. E. Gray et al. (McGraw-Hill, New York, 1963).

\title{
Energy of Excess Electrons in Nonpolar Liquids by Photoelectric Work Function Measurements*
}

\author{
R. A. Holroyd and Mark Atren \\ Chemistry Department, Brookhaven National Laboratory, Upton, Long Island, New York 11973
}

(Received 11 January 1971)

\begin{abstract}
The energy of the excess electron state has been determined for six nonpolar liquids by a photoelectric injection method. The functional dependence of the photocurrents on wavelength is the same in the liquid as in the vacuum and work functions can be evaluated from Fowler plots. For several liquids the work functions are less than the vacuum value and these lowerings are characteristic for each liquid. For tetramethylsilane, neopentane, cyclopentane, and 2,2,4-trimethylpentane the work functions are lowered by $0.62,0.43,0.28$, and $0.18 \mathrm{eV}$, respectively. For $n$-pentane and $n$-hexane the work functions are close to the vacuum value. The magnitude of the lowering is correlated with the energy of the first electronic absorption band. A variation of electron mobility with the work function changes is noted and discussed relative to the trap theory. The photocurrents measured in the liquids increase with voltage, and at the voltage employed the currents in neopentane and tetramethylsilane are comparable to the vacuum photocurrent. The magnitude of the observed photocurrent depends on the distance the electrons penetrate.
\end{abstract}

\section{INTRODUCTION}

Several recent studies ${ }^{1-3}$ have been published in which the mobility of electrons is reported for some nonpolar liquids. The values of the mobility are different for each liquid and range from a low value of 0.09 for $n$-hexane to $55 \mathrm{~cm}^{2} \mathrm{~V}^{-1} \cdot \mathrm{sec}^{-1}$ for neopentane. Since these values are at least two orders of magnitude larger than ionic mobilities, the electron is considered to be quasifree but inhibited in its motion either by scattering interactions or by trapping for short times at various sites in the liquid..$^{1,4}$ Aside from the mobility data, little is known about excess electrons in hydrocarbon liquids and a motivation of this study was to provide more information concerning this interesting state.

The properties of the excess electron state are better understood for liquid rare gases and hydrogen. The ground state energies of the electron have been determined by photoinjection work function measurements and are $+1.0 \mathrm{eV}$ in liquid $\mathrm{He},{ }^{5}-0.33 \mathrm{eV}$ in liquid $\mathrm{Ar},{ }^{6 \mathrm{a}}$ and $\leq 0.5 \mathrm{eV}$ in liquid $\mathrm{H}_{2}{ }^{6 \mathrm{~b}}$ The electron mobilities (in units of centimeters ${ }^{2}$ volts $^{-1} \cdot$ second $^{-1}$ ) are 0.02 in liquid $\mathrm{He}^{7} 0.03$ in liquid $\mathrm{H}_{2}{ }^{6 \mathrm{~b}}$ and 440 in liquid $\mathrm{Ar}^{8}$ The positive energy for helium is a consequence of the small polarizability of helium which results in the electron being localized in a cavity of $15-20 \AA$ radius. Liquid argon has a large polarizability and the electron is more stable in the liquid than in vacuum and localization does not occur. ${ }^{9}$ Since the electron mobilities observed for hydrocarbons vary over a range which is between the mobilities in helium and argon, it was anticipated that the ground state energies of electrons in hydrocarbons would also vary, and would increase with decreasing mobility. These energies have been determined for $n$-pentane, $n$-hexane, cyclopentane, 2,2,4-trimethylpentane, neopentane, and tetramethylsilane. The method used involved measuring the work function from the wavelength dependence of the photocurrent for various metals immersed in each liquid.

\section{EXPERIMENTAI}

The experiments consisted of determining apparent work functions of metal surfaces immersed in various liquids as well as the work functions of the same metal surfaces in vacuum. ${ }^{10 a}$ The difference for any one metal surface is denoted by $V_{0}$,

$$
V_{0}=\Delta \phi=\phi_{\mathrm{liq}}-\phi_{\mathrm{vac}}
$$

which defines the ground state of an electron in each liquid.

The photoelectric effect was used to determine work functions. The current, $i$, observed from a phototube can be expressed by

$$
i=\alpha A T^{2} F(x),
$$

